\title{
A MATHEMATICAL ALGORITHM FOR ECG SIGNAL DENOISING USING WINDOW ANALYSIS
}

\author{
Hamid SadAbadia*, Masood Ghasemi ${ }^{\mathrm{a}}$, Ali Ghaffari ${ }^{\mathrm{a}}$ \\ a CardioVascular Research Group (CVRG), Department of Mechanical Engineering, K. N. Toosi University of Technology, \\ No. 15, Pardis St., MollaSarda Ave., Vanak sq., Tehran, Iran, P. O. Box 19395-1999 \\ * corresponding author, graduate student of Mechanical Engineering, Tel: (+98) 21 8867-4841, Fax: (+98) 21 8867-4748 \\ e-mail: hamidsadabadi@sina.kntu.ac.ir
}

Received: December 17, 2006; Accepted: March 20, 2007

Key words: Electrocardiogram (ECG)/Window analysis/ECG denoising/Signal processing/Dominant scale

Background: The presence of parasite interference signals could cause serious problems in the registration of ECG signals and many works have been done to suppress electromyogram (EMG) artifacts noises and disturbances from electrocardiogram (ECG). Recently, new developed techniques based on global and local transforms have become popular such as wavelet shrinkage approaches (1995) and time-frequency dependent threshold (1998). Moreover, other techniques such as artificial neural networks (2003), energy thresholding and Gaussian kernels (2006) are used to improve previous works. This review summarizes windowed techniques of the concerned issue.

Methods and Results: We conducted a mathematical method based on two sets of information, which are dominant scale of QRS complexes and their domain. The task is proposed by using a varying-length window that is moving over the whole signals. Both the high frequency (noise) and low frequency (base-line wandering) removal tasks are evaluated for manually corrupted ECG signals and are validated for actual recorded ECG signals.

Conclusions: Although, the simplicity of the method, fast implementation, and preservation of characteristics of ECG waves represent it as a suitable algorithm, there may be some difficulties due to pre-stage detection of QRS complexes and specification of algorithm's parameters for varying morphology cases.

\section{INTRODUCTION}

The presence of parasite interference signals could cause serious problems in the registration of ECG signals. Most common problems are power line interference, electromyogram (EMG) or myopotential signals, motion artifacts, and baseline (drift) interferences ${ }^{1}$.

In practice, wideband myopotentails from pectoral muscle contraction will cause a noisy overlay with the ECG signal. It can be presented as:

$$
\text { Observed signal }=\mathrm{ECG}+\text { myopotential }
$$

In the above equation, the myopotential component of a signal corresponds to additive noise, thus obtaining the true ECG signal from noisy observations can be formulated as the problem of signal estimation or signal denoising ${ }^{2}$.

Fig. 1 shows two actual recorded ECG signals. One of them has very low rate noises, and the other consists of high rate noises. It is clear that the precise detection of onset and offset of its main waves in the noisy signal becomes very hard. Note that the sampling rate of the signals is $1 \mathrm{kHz}$ (According to the PTB diagnosis ECG database for Physionet/Challenge 2006)

While there are well-developed methods for power line, interference, and drift suppression, there are still problems in myopotential signal suppression due to the considerable overlapping of the frequency spectra of both types of signals. Thus, the automatic interpretation, following accurate detection of characteristic ECG points and waves such as $P$ wave, T wave, QRS complex and the measurement of signal parameters become an extremely difficult, sometimes virtually impossible task ${ }^{2}$.

Generally, adequate ECG denoising algorithms and procedures should have the following properties ${ }^{1}$ :

a) Improve signal-to-noise ratio (SNR) for obtaining clean and readily observable recordings, yielding the subsequent use of straightforward approaches for accurate automatic detection of characteristic points in the ECG signal and recognition of its specific waves and complexes

b) Preserve the original shape of the signal and especially the sharp Q, R, and S peaks, without distorting the $\mathrm{P}$ and $\mathrm{T}$ waves and the smooth transition of the ST-T segment.

Recently some new techniques based on global and local transforms have become popular in connection with signal denoising. At the first step, the signal is decomposed into a transform domain where filtering procedures are applied. The noise-free signal is then obtained by an inverse transform. Choosing appropriate basis functions for successful decorrelation of the signal and designing transform domain filters accommodated to the ECG sig- 

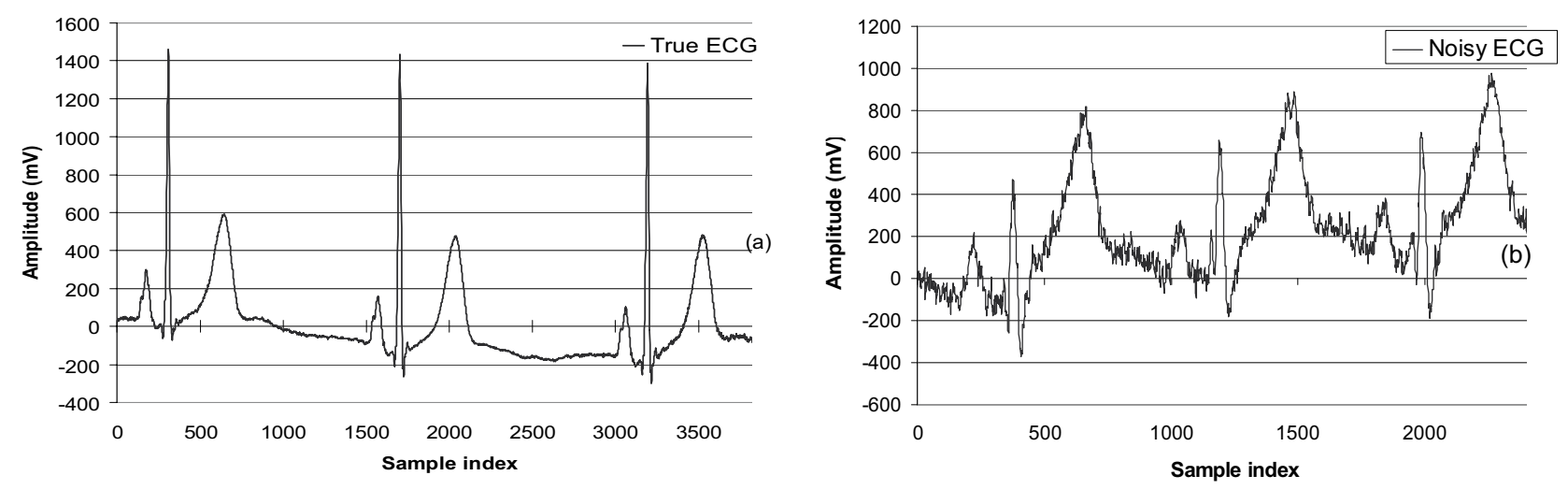

Fig. 1. Two ECG signals, a) True ECG signal with low noise rate; b) ECG signal with high noise rate. (The PTB Diagnostic ECG Database (MIT), Physionet/Challenge 2006(ref. $\left.{ }^{7}\right)$ ).

nal morphology could turn these techniques into powerful means for ECG signal denoising ${ }^{1}$.

A method for ECG denoising has been proposed based on the wavelet shrinkage approach ${ }^{3}$ using time-frequency dependent threshold (TFDT) (ref. ${ }^{4}$ ). The TFDT is high for non-informative wavelet coefficients, and low for informative coefficients representing the important signal features. Although giving relatively good results in comparison with other ECG denoising methods, the latter has some disadvantages: Some oscillations may occur at the end of the QRS-complexes using long-length decomposition filters due to the poor time localization of the basis functions; and in the opposite direction very short-length filters may corrupt the shapes of the "slow" $\mathrm{P}$ and $\mathrm{T}$ waves".

Using artificial neural networks (ANNs) for noise and baseline removal is also used in many articles; e.g., a neural network based adaptive algorithm for ECG denoising is presented in ${ }^{5}$. Furthermore, G.Clifford ${ }^{6}$ presented a general technique using energy thresholding and Gaussian kernels for biomedical signal denoising and he showed that significant noise reduction, compression, and turning point location is possible by this method. Also, there are many mathematically based algorithms using wavelet analysis to denoise ECGs and these can be found in many articles and papers.

Despite these mentioned algorithms, there are also problems in ECG denoising to preserve ECG morphology with a high degree of confidence. In the present study, we aim to improve the denoising procedure by using a mathematical algorithm.

The paper is organized as follows. Section 2 is the main part of the paper. It starts with a discussion about the mathematical concepts of QRS dominant scale and R-wave detection (subsections 2-1 and 2-2 respectively). In the third part of this section, the mathematical concepts of the proposed denoising algorithm are clarified in detail. The final part explains the proposed baseline-wandering algorithm. Section 3 has three parts. In the beginning, it discusses about the efficiency of this algorithm in denoising. In the second and third part of this section, the results of implementation of this algorithm are investigated in both a true ECG signal that is corrupted with additive noises manually and an actual recorded ECG signal respectively.

\section{MATERIALS AND METHOD}

The process of analyzing ECG signals with no prior information seems a lengthy process and needs advanced mathematical consideration. The prior information might consist of some special ECG characteristic points, HR, ECG waves bandwidth. In our algorithm, we use two extra sources of information, which are:

i. The QRS complex dominant scale, $a_{Q R S}$, is determined.

ii. Locations of R-waves in ECG signal are specified.

In addition, we assume noises cannot be observed in QRS complexes due to the high energy of the QRS complex compared with the low energy of the corrupting noises.

\section{2-1. Determination of QRS-complex dominant scale}

The duration of each component of ECG signal can be expressed as a function of time interval and the corresponding dominant rescaled wavelet coefficients. The following dominant scale for QRS complex is derived as below and has been proposed $\mathrm{in}^{8}$ :

$$
a_{Q R S}=1.00\left(\frac{D_{Q R S}}{T_{S}}\right)
$$

Where, $D_{Q R S}$ is the QRS complex duration and $T_{S}$ is rescaled wavelet coefficients.

While the width of the R-wave is slightly larger than the width of $\mathrm{Q}$ and $\mathrm{S}$ waves, the dominant scale of QRS complex does not change noticeably from that of R-wave. Thus, it is practical to use the evaluated dominant scale of the QRS complex for R-wave in the rescaled wavelet map ${ }^{9}$.

\section{2-2. $R$-Wave detection}

Based on the algorithm proposed ${ }^{9}$ in, in order to detect the R-wave vicinity, continuous wavelet transform of ECG signal is used. R-wave is more significant at its dominant scale; therefore, it is sufficient just to analyze the CWT of ECG signal at its R-wave dominant scale. The method is 
based on detection of high peaks primarily through which threshold search is used.

\section{2-3. Method for denoising the ECG signal}

It is possible to eliminate undesired high frequency components (noises) in the ECG signal using windowed analysis. By knowing the QRS complexes dominant scale and the position of every R-wave as mentioned before, a window is considered. The length of this window is defined as an integer function of QRS dominant scale, $a_{O R S}$, and location of every detected R-wave. (Equation (3)).

$$
d_{i}(x)=1+\left(\alpha \cdot a_{Q R S}-1\right) \times\left(1-e^{-\frac{1000 \beta \cdot\left(P_{i}-x\right)^{2}}{f \cdot a_{Q R S}}}\right)
$$

Where, $\alpha$ and $\beta$ are constant parameters, $P_{i}$ is the sample index of $i^{\text {th }}$ detected R-wave, $f$ is sample frequency, and $x$ is the sample index, which varies through:

$$
\frac{\left(P_{i-1}+P_{i}\right)}{2} \leq x \leq \frac{\left(P_{i}+P_{i+1}\right)}{2}
$$

The formula defines window length thorough the ECG signal. The length of this window varies from 1 at the location of every detected R-wave and increases until it reaches its maximum value, $\alpha \cdot a_{Q R S}$, between two detected R-waves as is shown in Fig. 2.

To remove noises, denoising window slides through the noisy signal and the value of the signal at the center of the window is set to the mean value throughout the window. If an ECG signal is represented as a discrete function $y(x)$, which $x$ denotes sample index, then the consequent denoised signal, $\bar{y}(x)$, at the $x^{\text {th }}$ sample index is obtained as follows:

$$
\bar{y}(x)=\frac{1}{d_{i}(x)} \sum_{j=-\frac{d_{i}(x)}{2}+x}^{\frac{d_{i}(x)}{2}+x} y(j)
$$

In this approach, the lowest value of window length, $d_{i}(x)=1$, which occurs in the location of every detected

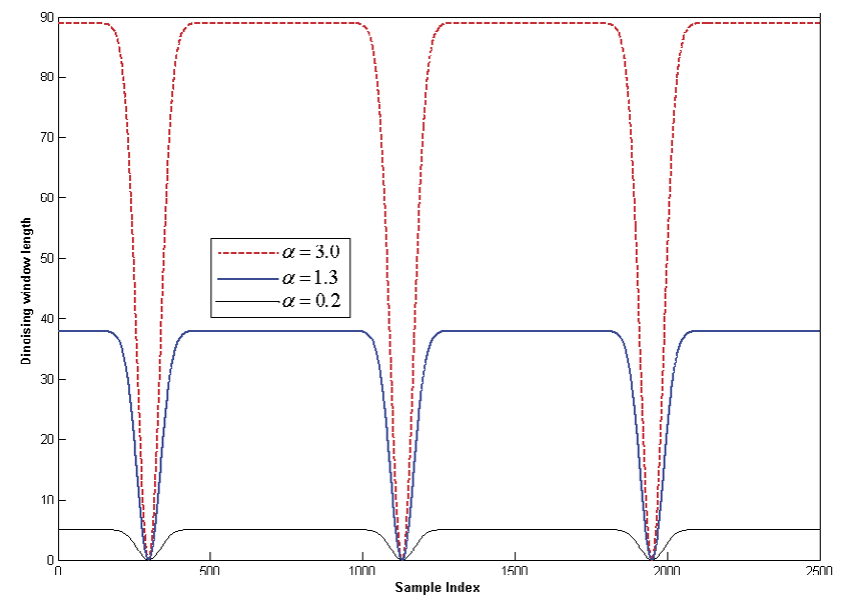

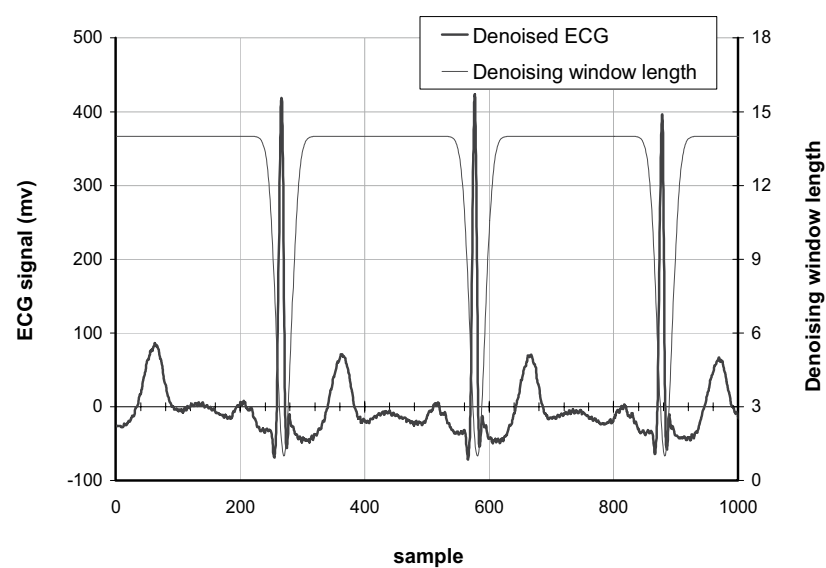

Fig. 2. Variation of denoising window through a sample ECG signal ${ }^{7}$ (R-waves detected at sample indexes 268, 580 and 880 respectively).

R-wave, produces almost original signal in the vicinity of QRS complex. In other words, a low length denoising window passes over QRS complexes vicinity and therefore, a slight denoising process is held on QRS complexes. The length of averaging window reaches its maximum between two successive detected $\mathrm{R}$-waves. This maximum is equal to $\alpha \cdot a_{Q R S}$ which results in rough noise removal in this region.

Fig. 2 illustrates variation of the length of denoising window (averaging window) through a sample signal that we would like to denoise it. The locations of detected R-waves are at sample indexes 268, 580, and 880 respectively.

Performance of denoising process strictly depends on window parameters, $\alpha$ and $\beta$. Parameter $\alpha$ determines maximum denoising window length while $\beta$ determines window's growth rate around the detected R-wave.

High values of $\alpha$ cause extensive noise removal between two successive QRS complexes, which is desirable for rich noisy signals. In contrast, low $\alpha$ values cause slight or weak noise removal between two successive QRS com-

Fig. 3. The behavior of window length function with respect to $\alpha$ and $\beta$ changes:

(a) the value of $\beta=0.02$ is held constant and $\alpha$ changes

(b) the value of $\alpha=1.3$ is held constant and $\beta$ changes.

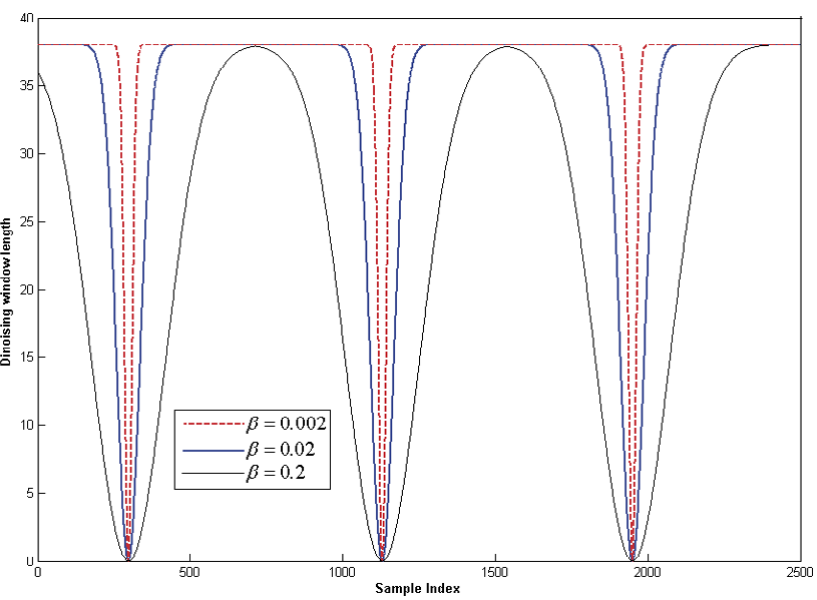




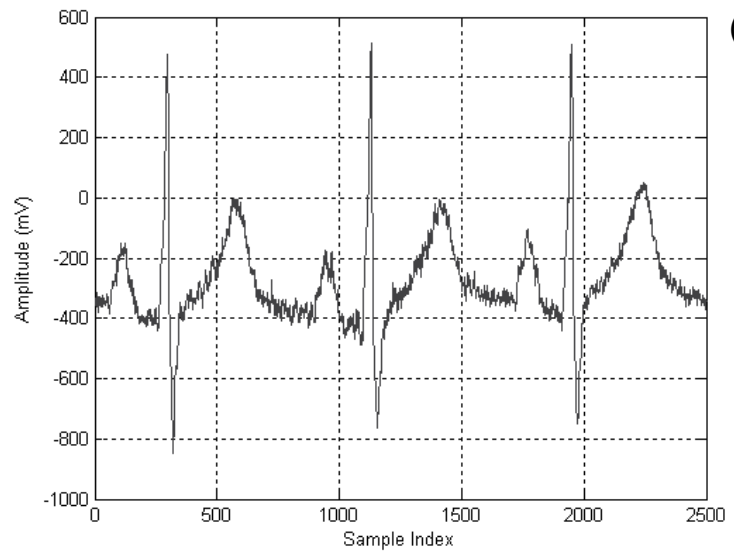

(a)

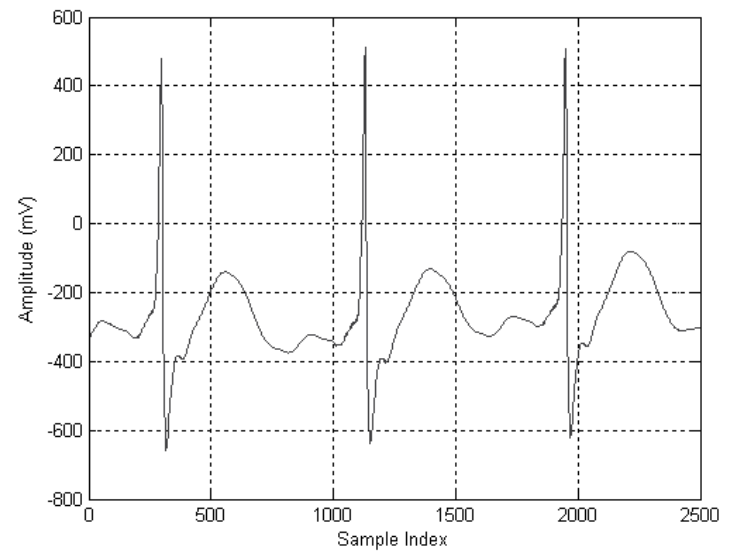

(b)
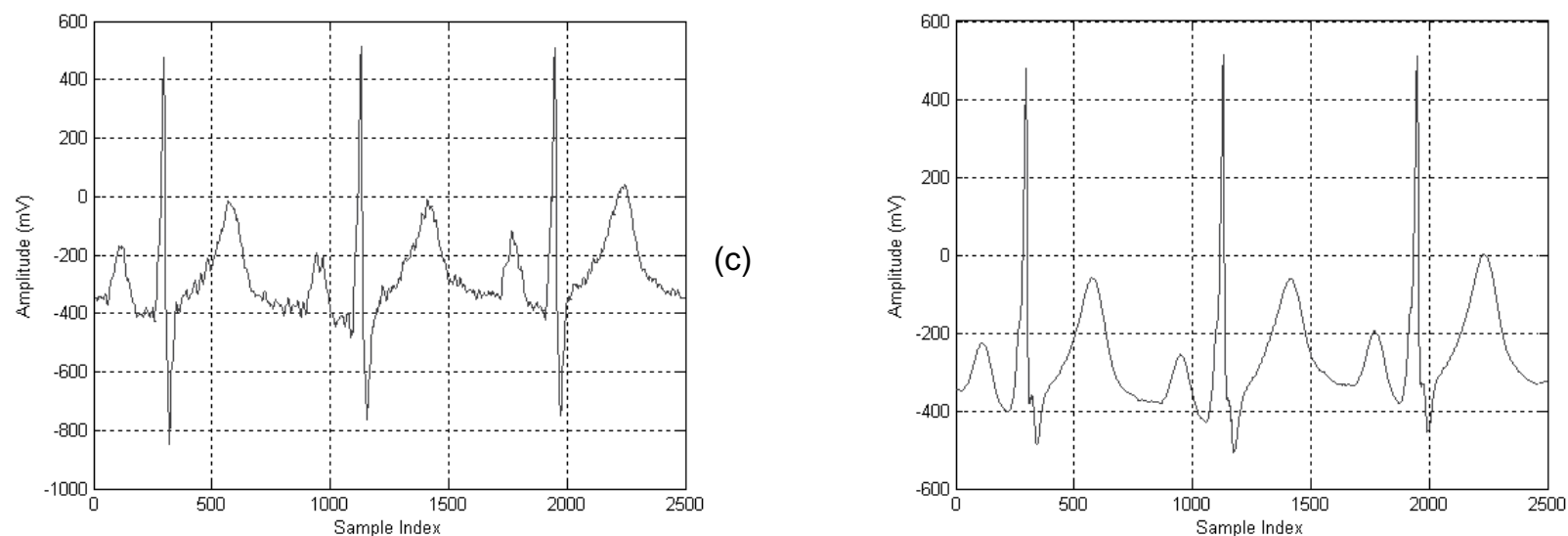

(d)
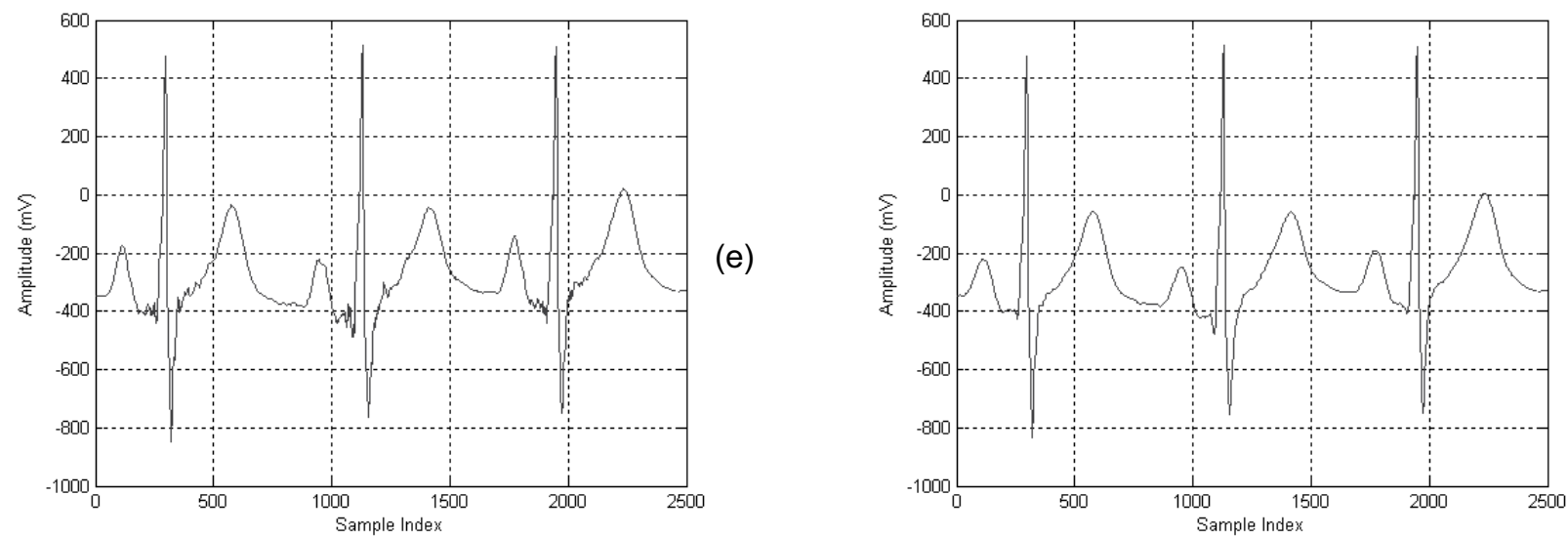

Fig. 4. Effect of parameters of denoising window $\alpha$ and $\beta$ on noise removal process ${ }^{7}$;

a) original noisy ECG signal, b) $\alpha=3.0 \& \beta=0.2$, c) $\alpha=0.2 \& \beta=0.02$, d) $\alpha=1.3 \& \beta=0.2$, e) $\alpha=1.3 \&$ $\beta=0.002$, f) $\alpha=1.3 \& \beta=0.02$.

plexes, which is desirable for slightly noisy signals. Low $\beta$ values cause low rate noise removal at the vicinity of QRS complexes, which is adequate for prolonged QRS morphologies. In contrast, high values of $\beta$ cause high noise rate removal around $\mathrm{QRS}$ complexes, which might deform QRS morphologies.

For rather normal range of QRS-complex duration and normal rate of noise interference, optimums values of $\alpha$ and $\beta$ are around 1.3 and 0.02 respectively.
Figs. 3-a and 3-b show the variation of window length related to changes in $\alpha$ and $\beta$. In Figure 3-a, the variation is depicted in which $\beta$ is constant and equals to $\beta=0.02$, and $\alpha$ gets three different values, $0.2,1.3$, and 3.0 alternatively. In Figure 3-b, the variation is depicted in which $\alpha$ is constant and equals $\alpha=1.3$, and $\beta$ gets three different values, $0.002,0.02$, and 0.2 alternatively. It is assumed that detected R-waves are located at sample indexes 298 , 1130 , and 1950 respectively. 


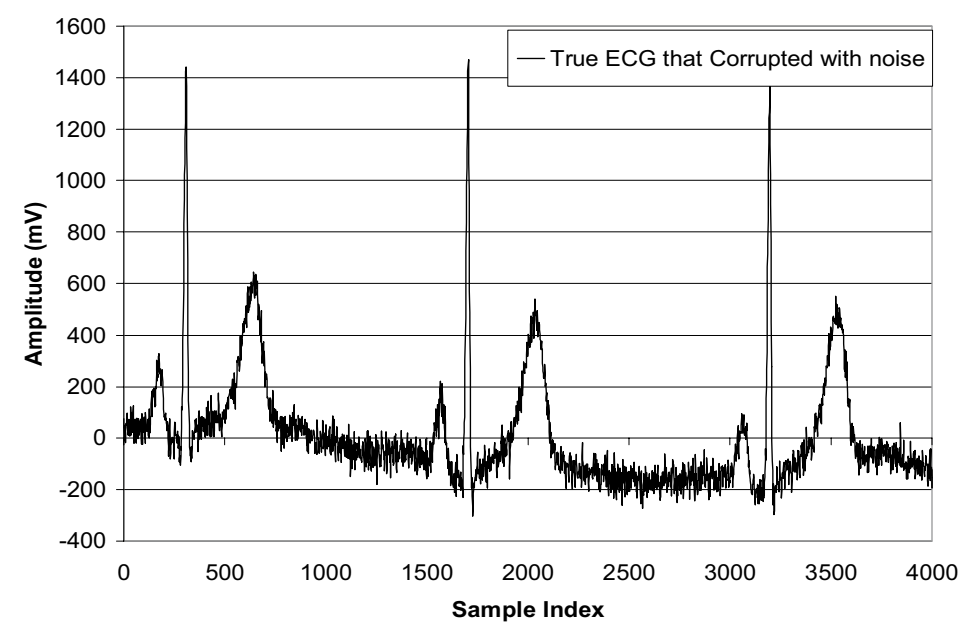

(a)

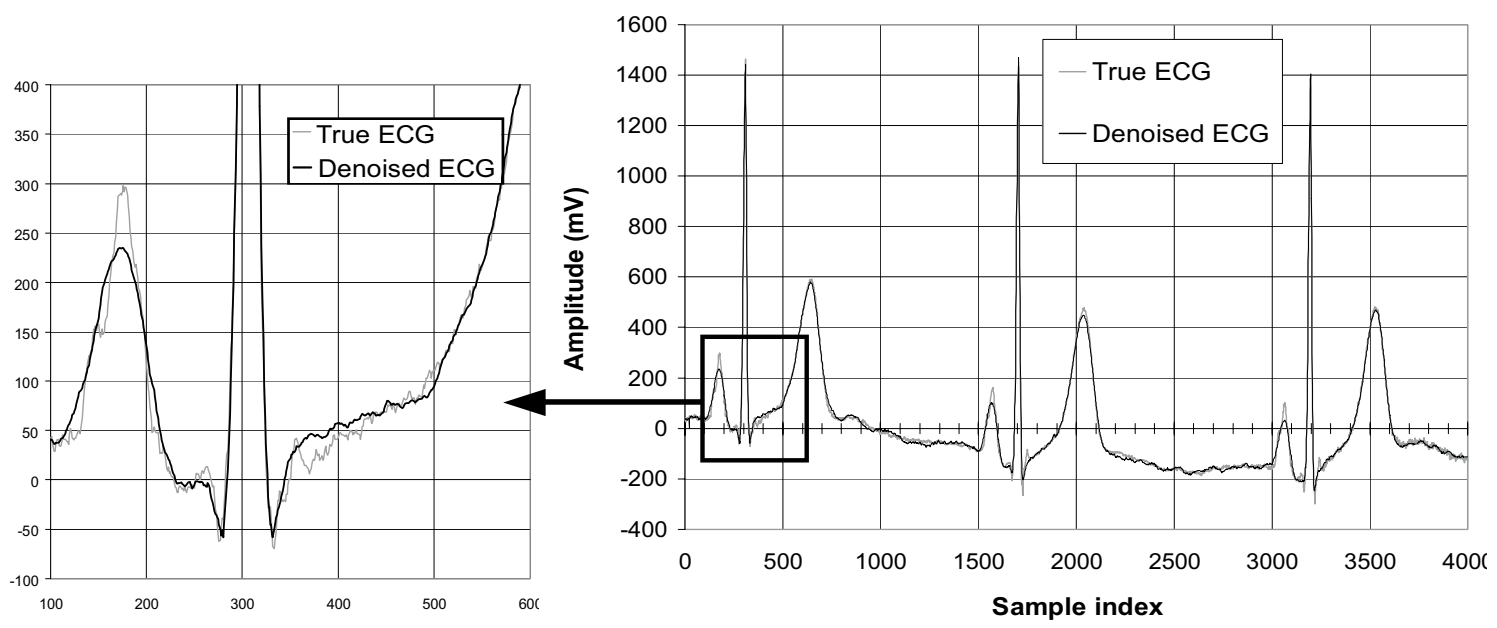

(b)

Fig. 5. Performance of denoising assessment on manually corrupted noised ECG signal; a) recorded noisy ECG signal, b) original signal compared with denoised one (The data are from MIT-PTB Database, Physionet/Challenge 2006( (ef. $\left.\left.^{7}\right)\right)$.

\section{2-4. Method for baseline wandering removal}

Another problem in ECG signal denoising task is baseline wandering. In this regard, we follow a slightly similar method, so as to a window analysis is proposed to remove baseline wandering. We consider a constant length for this window which equals two seconds, for instance, if sample frequency of signal, $f$, is equal to $1000 \mathrm{~Hz}$, then the length of the window is 2000 sample. Then the main value through this window is calculated (Equation (6)).

$$
\tilde{y}(x)=\sum_{j=x-f}^{x+f-1} \frac{y(j)}{2 . f}
$$

Baseline wandering is removed by subtracting the calculated mean value, $\tilde{y}(x)$ from ECG signal. Considering equations 5 and 6 , both noiss and baseline wandering are eliminated simultaneously, the consequent formula can be expressed as Equation 7.

$$
Y(x)=\bar{y}(x)-\tilde{y}(x)
$$

Where, $Y(x)$ is the final resulting signal while it is mainly freed from noise and baseline wandering interference.

\section{RESULTS AND DISCUSSION}

\section{3-1. Discussion of the effects of Parameters $\alpha$ and $\beta$ on denoising efficiency}

Fig. 4-a shows a recorded noisy ECG signal that we would like to denoise. Figs. 4-b and 4-c show the denoising process with unsuitable high and low values respectively for parameter $\alpha$, which caused rough and inadequate noise removal. Furthermore, Figs. 4-d and 4-e show denoising process with unsuitable high and low values for parameter $\beta$ respectively. Note that QRS complex morphology deformation in Fig. 4-d and inadequate denoising in the vicinity of the QRS complexes in Fig. 4-e is obvious.

By choosing optimum values for these two parameters we can have both adequate denoising and ECG morphology preservation that is shown in Fig. 4-f. 


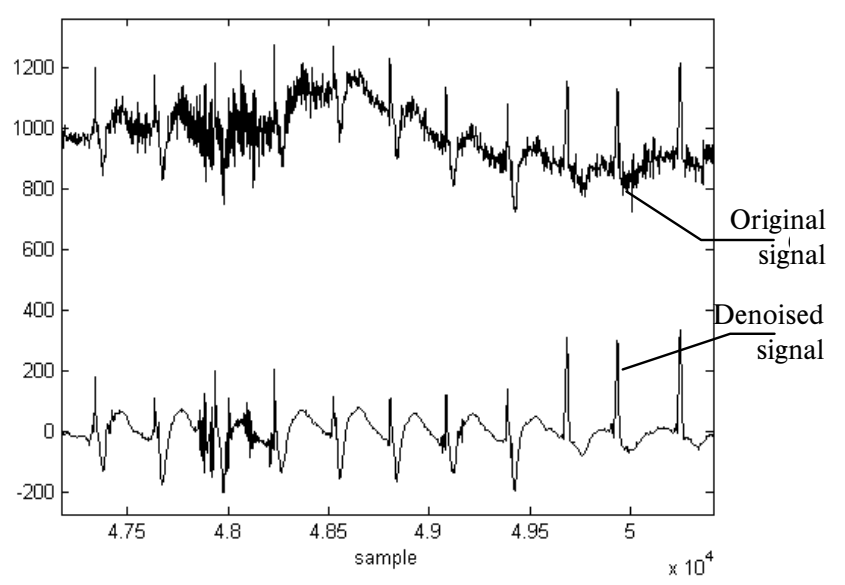

Fig. 6. Noisy ECG signal and its Denoised ECG signal with removed baseline wandering (MIT-BIH database, patient 104).

\section{3-2. Denoising algorithm Performance Assessment}

In order to guarantee the performance of the proposed denoising algorithm, we implemented this method on a true ECG signal that is corrupted manually with additive noises (Fig. 5-a).

Fig. 5-b shows the actual signal and the denoised one. We can see that the denoised signal morphologically is most similar to its original signal. On the other hand, the main waves of ECG signal still maintain their basic morphologies. This is observable in all $\mathrm{P}$ and $\mathrm{T}$ waves and QRS complexes.

\section{3-3. Empirical Results}

In order to validate the proposed algorithm, we implemented the method on noisy recorded ECG signals. An empirical result is shown in Fig. 6. It is clearly seen that noise removal process with acceptable performance is held while its main morphologies are kept. Baseline wandering is also removed by considering local averages over two seconds of ECG signal as mentioned before.

A slight malfunctioning of the method around sample $4.8 \times 10^{4}$ is because of $\mathrm{R}$-wave detection fault and this is because of the fairly high value of noises in that region.

\section{CONCLUSION}

A simple mathematical algorithm for suppressing of noises from ECG signal is proposed in this paper. The advantages of this four-stage algorithm can describe as:
- Very fast algorithm for ECG denoising

- Mathematically simple algorithm

- Preserve QRS complex characteristic points, especially $\mathrm{Q}$ and $\mathrm{S}$ waves.

Despite above advantages, there are still some limitations in the denoising stage. Those limitations will appear if:

- The morphology of QRS complex is a prolonged one with rather small dominant scale.

- Pre-stage detection of R-waves fails.

- The smoothness and morphology preservation of denoised ECG signals strongly depend on the selected values for parameters $\alpha$ and $\beta$.

\section{ACKNOWLEDGMENTS}

The authors would like to express their deep gratitude to Prof. George Moody from Harvard-MIT division of Health and Science (HST) for his interest in this project and the Physionet/Challenge, PTB Diagnostic ECG signal database and MIT-BIH database.

\section{REFERENCES}

1. Nikolay Nikolaev, Atanas Gotchev. ECG Signal Denoising Using Wavelet Domain Wiener Filtering, Proceeding of the European Association for Signal and Image Processing (EURASIP), 2000.

2. Vladimir Cherkassky, Steven Kilts. Myopotential denoising of ECG signals using wavelet thresholding methods, contributed article. Neural Networks, Elsevier 2001; 14(8):1129-1137.

3. D. L. Donoho and I. Johnstone. Adapting to unknown smoothness via wavelet shrinkage. Amer. Stat. Assoc. 1995; 90:1200-1224.

4. N. Nikolaev, A. Gotchev. De-noising of ECG signals using wavelet shrinkage with time-frequency dependant threshold, Proceedings of the European Signal Processing Conf. EUSIPCO-98, Island of Rhodes, Greece; September 1998, pp. 2449-2453.

5. M.H. Sedaaghi and E. Ajami. Neural-Network-based Adaptive ECG Denoising, Proceeding (385) of Artificial Intelligence and Soft Computing (ASC), Banff, Canada, 2003.

6. Gari D. Clifford. (2006) A Novel Framework For Signal Representation and Source Separation: Application to filtering and segmentation of Biosignals. Biological Systems 2006; 14(2):169183.

7. Hans Koch, Prof., Ph.D, Lothar Schmitz, M.D. The PTB Diagnostic ECG Database, PhysioNet Library, MIT Room E25-505A.

8. A. Ghaffari, H. Golbayani, M. Ghasemi. (2006) Mathematical based algorithm for QRS complex detection using continuous wavelet transform, (preliminary accepted, under revision) journal of computers and electrical engineering, 2006, Elsevier.

9. M. Ghasemi, H. DadAbadi, H. Golbayami, A, Ghaffari. ECG characteristic point detection using cantinuous wavelet transform, submited to J Biomed Sig Proc Contr., 2007, Elsevier. 